\title{
The influence of profile angle on forces distribution on silent chain transmissions
}

\author{
Radu Velicu $^{1 *}$, Lenard Jurj ${ }^{1}$ and Radu Săulescu ${ }^{1}$ \\ ${ }^{1}$ Transilvania University of Brasov, Product Design Mechatronics and Environment Department, Eroilor 29, 500069 Brasov, Romania
}

\begin{abstract}
The aim of this paper is to define and to analyze the theoretical contact forces that appear in a sprocket - silent chain joint. The contact force between the links and the teeth flanks of the sprocket can be considered as a reaction to the axial force (tensioning force) transmitted throughout the pins. The centrifugal force is considered to be constant and the friction between the elements is not considered. This paper is presenting the steps to obtain the relationships for the forces from the sprocket-link contact depending on the tensioning force component between the links and the centrifugal force. These forces are computed in order to establish their distribution on links.
\end{abstract}

\section{Introduction}

Chain drive system's dynamics became of great interest to the automobile industry with the increase in importance of noise, vibration, and harshness (NVH noise, vibration and harshness). Chain drives are used because of the non-slip, reliability and compact power transmission compared to belt transmissions, but usually with the disadvantages of increased noise and vibration. A brief history of chain drive systems and the important milestones in their practical use and development through the 20th century can be found in [1].

Timing chain transmissions are used more and more in combustion engines. Their robustness ensures the transmission of higher power, respectively higher torque for a longer maintenance-free period aggregate, [2].

Silent chains as timing chains have a great interest regarding performance improvements of automobile engine systems, which can generate benefits like: reduced consumption of fuel and oil, higher engine power and torque, reduced harmful gas emissions, longer life and improved reliability of engines [3]-[5].

Silent chains are usually composed, as seen in Figure 1. of: 1 - inner plates, 2 - middle plates, 3 - outer plates and $4-$ pin. The inner plates and the outer plates are fixed on the pin, while the middle plates are mounted with clearance on the pins. Only the inner and the middle plates are engaging with the teeth of sprockets. The outer plates are used for guiding. This type of chain is not standardized, there are different shapes and sizes of the plates; different number of inner and middle plates.

References of theoretical studies on silent chain transmissions come from early 20th century, [6]. A geometrical study of chain transmissions with two sprockets and a guide is presented in [7]. An FEM study of forces distribution on links of a silent chain, at the contact with a circular guide, is presented in [8]; pressure distribution between a silent chain and a guide, resulted from a tribological study, is presented in [9]. A comprehensive approach on general chain transmissions, including aspect of silent chains is in [10]; experimental results on load distribution between bush chains and sprockets is presented in [11]. This paper intends to study the theoretical contact which appears between the silent chain's elements and the sprockets.

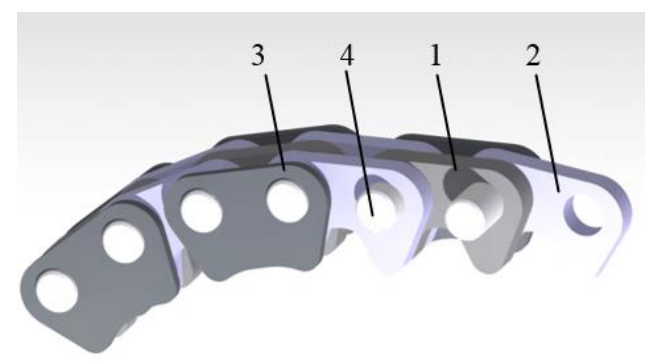

Fig. 1. Silent chain.

\section{Theoretical approach}

Figure 2. presents the theoretical model of the silent chain transmission. The middle or the inner plates of each link are coming in contact directly with the linear flank of the tooth, without rolling or sliding.

$F$ is the tensioning force applied to the first pin on the first link (1) of the chain. The theoretical force distribution takes into account the normal forces that appear between the sprocket tooth and chain plate of link $1-F_{z l}$, the tensioning force component between the silent chain links 1 and 2, that is transmitted by the second plate-pin joint $F_{l}$, and the centrifugal force acting on link 1 that appear during the rotational speed of the chain transmission $-F_{c}$.

\footnotetext{
* Corresponding author: rvelicu@unitbv.ro
} 


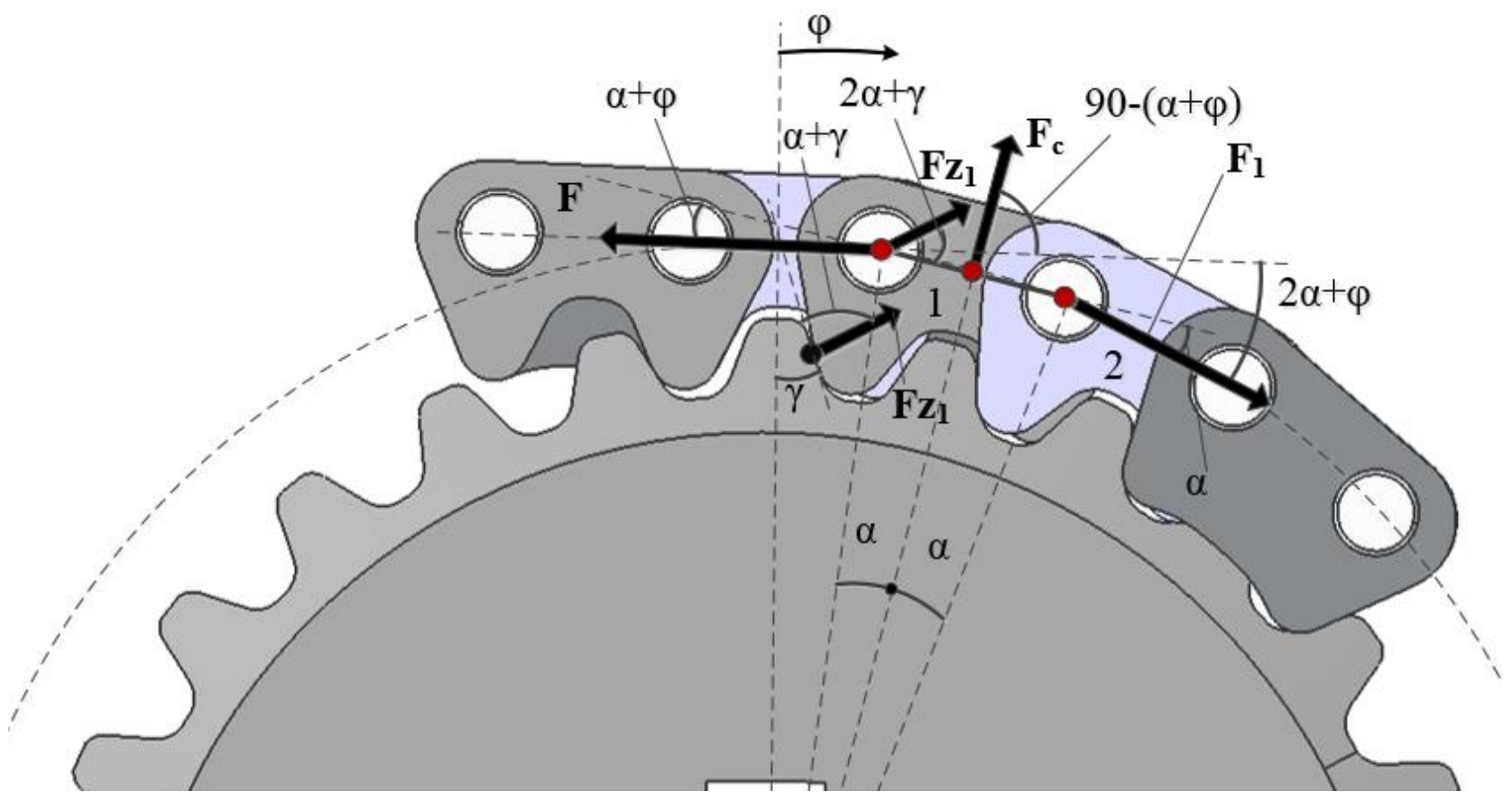

Fig. 2. Force distribution on silent chain transmission.

In this case, the force distribution is varying within an angle $\varphi$ from $-\alpha$ to $\alpha$, where $\alpha$ represents the half of the pitch angle on the sprocket. This angle depends on the number of teeth of the sprocket. The profile angle $\gamma$ appears between the axis of a tooth and its flank line (plate's exterior flank). There will be taken into account three values for $\gamma$ to find out what the effects of this angle are over the overall force transmission between the sprocket and the chain.

The theoretical model will be established using a constant rotational speed of the system, thus constant centrifugal force on the plates.

The calculus starts with the equilibrium of the forces written on two directions:

- $\quad$ on the direction of the tensioning force $F$ :

$$
\begin{aligned}
& F=F_{\mathrm{z} 1} \cos (\alpha+\gamma-\varphi)+F_{\mathrm{c}} \sin (\varphi+\alpha)+; \\
& +F_{1} \cos (\varphi+2 \alpha)
\end{aligned}
$$

- on perpendicular direction over the tensioning force:

$$
\begin{aligned}
& F_{1} \sin (\varphi+2 \alpha)=F_{\mathrm{z} 1} \sin (\alpha+\gamma-\varphi)+ \\
& +F_{\mathrm{c}} \cos (\varphi+\alpha)
\end{aligned}
$$

Extracting from (2) the force from the plate-sprocket contact $F_{z I}$ we have:

$$
F_{\mathrm{z} 1}=\frac{F_{1} \sin (\varphi+2 \alpha)-F_{\mathrm{c}} \cos (\varphi+\alpha)}{\sin (\alpha+\gamma-\varphi)},
$$

Using (3) in (1) we get the equation for the component of the tensioning force between the chain links:

$$
F_{1}=\frac{F \sin (\alpha+\gamma-\varphi)+F_{\mathrm{c}} \cos (2 \alpha+\gamma)}{\sin (3 \alpha+\gamma)}
$$

Based on Figure 2, the following equations can be drawn:

$$
\begin{aligned}
& \alpha=\frac{360}{2 z} \\
& v_{\mathrm{t}}=\frac{\pi \cdot D_{\mathrm{p}} \cdot n}{1000 \cdot 60} \\
& F_{\mathrm{c}}=\frac{m_{\text {chain }} \cdot v_{\mathrm{t}}^{2}}{\frac{D_{\mathrm{p}}}{2} \cdot 1000}
\end{aligned}
$$

where $z$ is the number of teeth on the sprocket, $D p$ is the pitch diameter of the sprocket, $\mathrm{n}$ is the rotational speed of the chain transmission, $v_{t}$ is the tangential speed at the pitch diameter and $m_{\text {chain }}$ is the mass of the silent chain.

For the equilibrium of link 2, the input force is the tensioning coming from link $1 F_{l}$, which will replace the input $F$ force used for equilibrium of link 1 . The relative angle between the links 1 and 2 being constant, angle $\varphi$ in (3) and (4) will have the value of the pitch angle $\alpha$.

The force $\mathrm{F} 1$ for the second link will become $F_{2}$, while $F_{z 1}$ will become $F_{z 2}$. All these equivalent replacements of the forces will be computed with the following observation: $F_{l}$ and $F_{z l}$ are varying in function of the angle $\varphi$ of link 1.

The tensioning force component for the second link can be described as:

$$
F_{2}=\frac{F_{1} \sin (\gamma)+F_{\mathrm{c}} \cos (2 \alpha+\gamma)}{\sin (3 \alpha+\gamma)} .
$$

The forces acting between the sprocket and the second link can be written as: 


$$
F_{\mathrm{z} 2}=\frac{F_{2} \sin (3 \alpha)-F_{\mathrm{c}} \cos (2 \alpha)}{\sin (\gamma)} .
$$

For the next links, the forces can be computed with the following generalized formulas:

$$
\begin{aligned}
& F_{\mathrm{m}}=\frac{F_{\mathrm{m}-1} \sin (\gamma)+F_{\mathrm{c}} \cos (2 \alpha+\gamma)}{\sin (3 \alpha+\gamma)} \\
& F_{\mathrm{zm}}=\frac{F_{\mathrm{m}-1} \sin (3 \alpha)-F_{\mathrm{c}} \cos (2 \alpha)}{\sin (2 \gamma)}
\end{aligned}
$$

where $m$ represents the number of the corresponding link of the silent chain.

\section{Numerical analysis - Results}

The model for the force equilibrium presented above has been applied for $z=27$ and three values of profile angle $\gamma\left(20^{\circ}, 25^{\circ}, 30^{\circ}\right)$. Table 1 presents the input parameters used to compute the theoretical forces that appear between the silent chain and the sprocket, calculated according to [12].

Table 1. Gear parameters, $z=27, \gamma\left(20^{\circ}, 25^{\circ}, 30^{\circ}\right)$.

\begin{tabular}{|c|c|c|}
\hline Symbol & Unit & Value \\
\hline$z$ & - & 27 \\
\hline$n$ & $\mathrm{rpm}$ & 5000 \\
\hline$F$ & $\mathrm{~N}$ & 500 \\
\hline$\alpha$ & $\mathrm{deg}$ & 6.66666 \\
\hline$P$ & $\mathrm{~mm}$ & 8 \\
\hline$D_{\text {pitch }}$ & $\mathrm{mm}$ & 68.91032 \\
\hline$M_{\text {chain }}$ & $\mathrm{kg}$ & 0.0034 \\
\hline$L_{\text {chain }}$ & $\mathrm{mm}$ & 518 \\
\hline$v_{t}$ & $\mathrm{~mm} / \mathrm{s}$ & 9.51745 \\
\hline$F_{\text {c/plate }}$ & $\mathrm{N} / \mathrm{plate}$ & 5.082994 \\
\hline
\end{tabular}

The diagrams of tensioning force $F_{m}$ depending on rotation angle $\varphi$, for three values of the contact angle $\gamma$, are presented for links 1, 2 and 3 in Figure 3., Figure 4. and Figure 5. They are showing the continuous decrease with rotation angle and sequent number of links, with smaller values for smaller contact angle. The diagrams of contact forces $F_{z m}$ depending on rotation angle $\varphi$, for three values of the contact angle $\gamma$, are presented for links 1, 2 and 3 in Figure 6., Figure 7. and Figure 8. The contact force on the first contact increases with rotation angle and with decrease of contact angle, but for the following contacts there is a continuous decrease with rotation angle.

For the sequent number of links, the trend on the contact force is a faster decrease for smaller values of the contact angle. The decrease of contact angle determines a decrease of contact force starting with link 3 . The second link is just a transition situation between the trend of the first link and the trend of the next links.

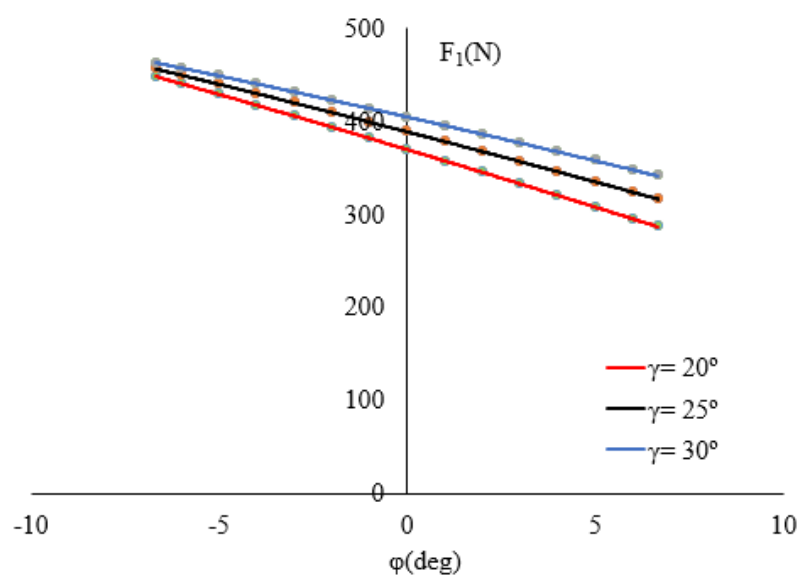

Fig. 3. Tensioning force distribution on the first link for three values of the profile angle $\gamma\left(20^{\circ}, 25^{\circ}, 30^{\circ}\right)$.

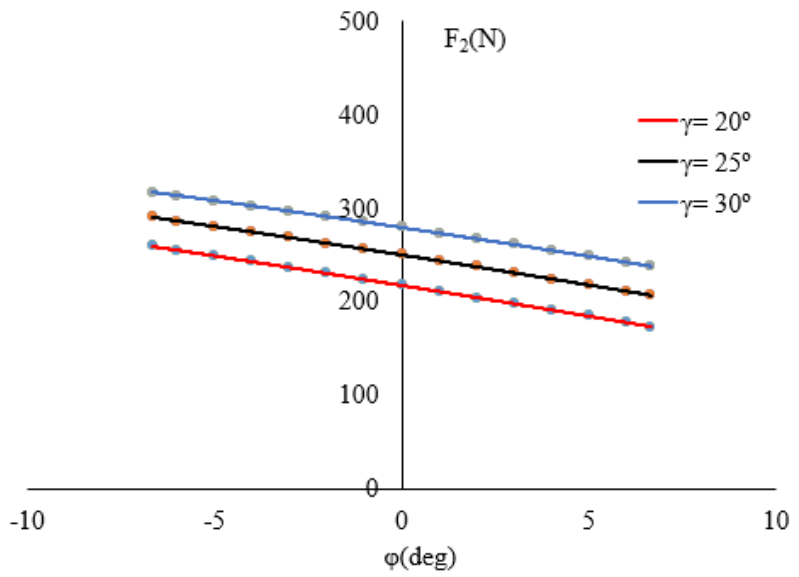

Fig. 4. Tensioning force distribution on the second link for three values of the profile angle $\gamma\left(20^{\circ}, 25^{\circ}, 30^{\circ}\right)$.

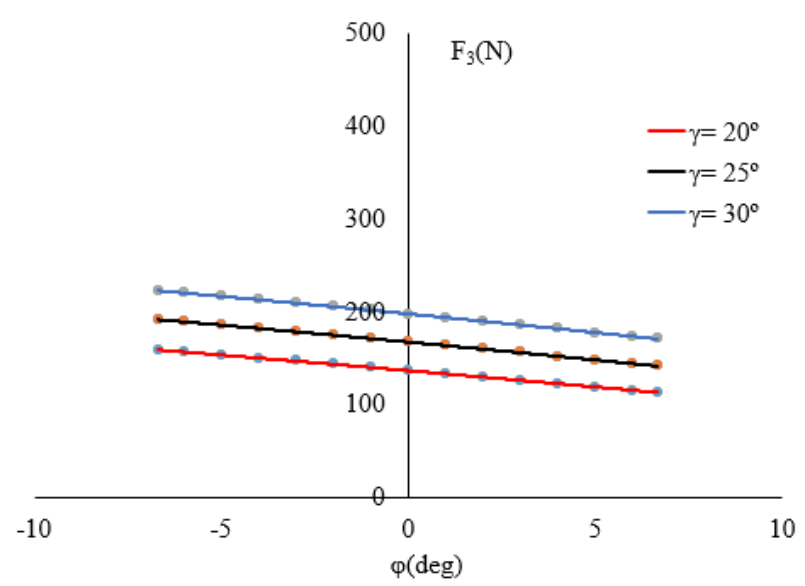

Fig. 5. Tensioning force distribution on the third link for three values of the profile angle $\gamma\left(20^{\circ}, 25^{\circ}, 30^{\circ}\right)$.

The analysis of the theoretical force distribution shows that the tensioning force in the silent chain transmission is distributed on more than the first couple of chain links. 


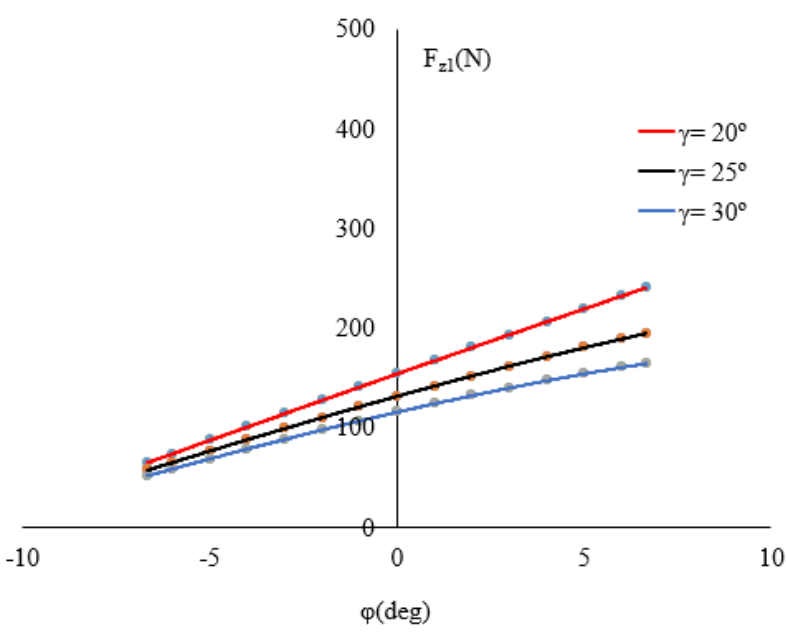

Fig. 6. The contact force on the first link for three values of the profile angle $\gamma\left(20^{\circ}, 25^{\circ}, 30^{\circ}\right)$.

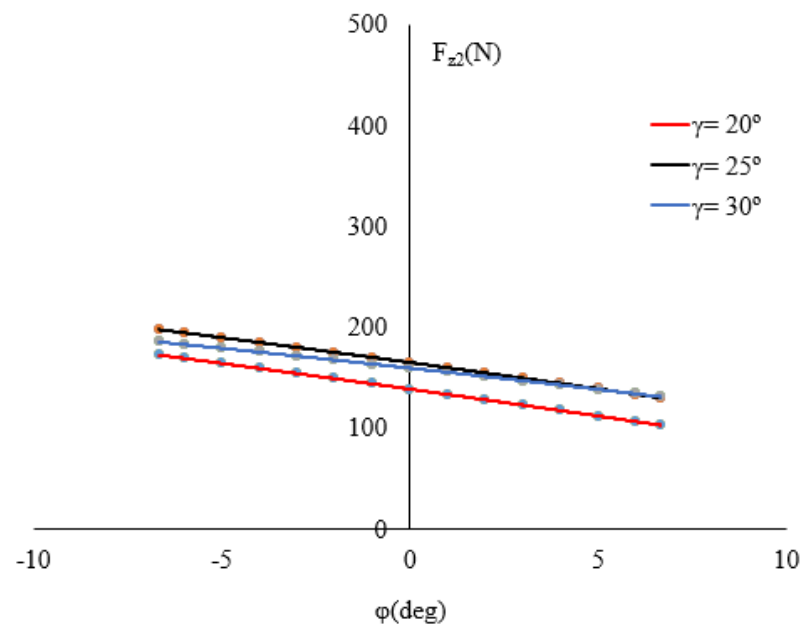

Fig. 7. The contact force distribution on the second link for three values of the profile angle $\gamma\left(20^{\circ}, 25^{\circ}, 30^{\circ}\right)$.

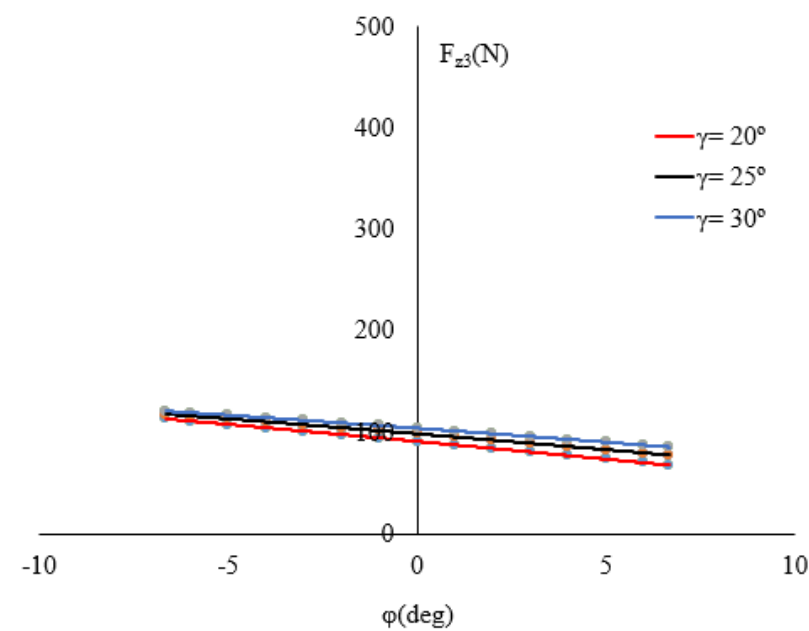

Fig. 8. The contact force distribution on the third link for three values of the profile angle $\gamma\left(20^{\circ}, 25^{\circ}, 30^{\circ}\right)$.
The results of the theoretic analysis for the silent chainsprocket shows that:

1) When the forces get to negative values the chain gets off the sprocket - until that moment the force are transmitted between the chain links and the sprocket.

2) The profile angle of the silent chain plate (middle plate, inner plate) has a great effect on the number contacts between the chain and the sprocket. As the profile angle increases the number of links in contact increases.

3) The $F_{z}$ component from the chain transmission, as a reaction from the sprocket to the chain link, tends to move off the links from the sprocket.

\section{Conclusion}

The main conclusion from theoretical analyse of the silent chain contact forces is that the number of chain links in contact is influenced by the profile angle of the tooth flank.

However, this conclusion must be proved when the friction forces are also considered.

\section{References}

1. J. C. Conwell, An Examination of Transient Forces in Roller Chain Drives, PhD Thesis, Vanderbilt University, Nashville (1989).

2. S. Belmer, T. Fink, , I. Lorenz, H. Neukirchner, MTZ 66, 6 (2005).

3. K. Holmberg, P. Andersson, A. Erdemir, Trib Int 47, (2012).

4. T. Hyakutake, M. Inagaki, M. Matsuda, N. Hakamada, Y. Teramachi, JSAE Review 22:5 (2001).

5. M. Schwaderlapp, F. Koch, J. Dohmen, FISITA (2000).

6. J. R. Cautley, SAE Technical Paper 140021 (1914).

7. R. Papuc, R. Velicu, C. C. Gavrila, Annals of the Oradea University, Fascicle of Management and Technological Engineering XXIII (XIII) (2014).

8. M. T. Lates, R. Papuc, C. C. Gavrila, Annals of the Oradea University, Fascicle of Management and Technological Engineering XV (XXV)/1 (2016).

9. R. Papuc, C. C. Gavrilă, M. Lates, Bulletin of the Transilvania University of Brasov 9 (58) 2 Prasic (2016).

10. J. H. Pfalzgraf, Experimental research on the chain transmission from timing system of combustion engines (in German), PhD thesis Technical University Munchen (2011).

11. M. R. Naji, K. Marshek, Journal of Mech., Trans. and Autom. in Design 105 (1983).

12. Schaeffler Group Automotive, SCDS Catalogue (2009). 